CLINICAL ETHICS

\title{
Ethical considerations in the application of preconditioning to solid organ transplantation
}

\author{
S J McNally, E M Harrison, S J Wigmore
}

J Med Ethics 2005;31:631-634. doi: 10.1136/jme.2004.011486

The shortage of organs for transplantation has led researchers to look for new techniques to expand the donor pool. Preconditioning strategies have the potential to protect organs from transplant associated injury or may improve the function of substandard organs so that they become suitable for transplantation. Translating this type of technology to the clinical setting raises ethical issues, particularly relating to the deceased donor. It is important that society has the opportunity to discuss the issues raised by implementation of preconditioning strategies before they are implemented rather than as a reaction to them.

O gan transplantation has been one of the great medical successes of our times. It has revolutionised the treatment of organ failure, often allowing the recipient to resume a normal lifestyle. ${ }^{1}$ Every advance has led to an increased demand for organs for transplantation and despite major improvements in immunosuppression, organ preservation, and operating techniques, patients continue to die on waiting lists due to the shortage of donors. ${ }^{2}$ The number of potential recipients in the United Kingdom awaiting organ transplantation has increased annually, reaching more than 7000 by March 2004. ${ }^{2}$ New sources of organs, such as living donors, split livers, and non-heartbeating donors, have increased available organs to a degree; however, these developments have failed to keep pace with the decline in the available number of cadaveric organs. Novel therapies such as stem cell technology and bioartificial livers hold promise for the future, but have not yet fulfilled their potential. There remains, therefore, a requirement for solid organ transplantation that is unlikely to recede in the near future.

Preconditioning is a developing technique, which is currently making the transition from an experimental concept to a practical therapeutic option. ${ }^{3}$ The basic premise is that treatment before a known injury occurs can be used to minimise the severity of that injury. This principle has widespread application in many areas of medicine, particularly in surgical disciplines. ${ }^{4}$ Solid organ transplantation, with its attendant ischaemia/ reperfusion injury, represents an area where preconditioning of the donor or the donated organs could make a great contribution, ${ }^{56}$ potentially reducing primary non-function and early graft failure, and thus the requirement for retransplantation. There is also experimental evidence that preconditioning may allow organs that would currently be discarded to be used, thus expanding the organ pool. In this paper we discuss the practical and ethical issues involved in implementing preconditioning strategies in solid organ transplantation.

\section{PRECONDITIONING}

\section{Practical implementation}

The term preconditioning encompasses many different techniques, which all rely on harnessing aspects of the innate protective mechanisms that human cells use to survive stress. Broadly speaking there are two approaches to induce preconditioning: physical and pharmacological. Physical techniques include ischaemic preconditioning ${ }^{7}$ and heat treatment. ${ }^{89}$ Pharmacological techniques include the administration of drugs, cytokines, and gene transfer techniques. ${ }^{10-12}$

Pharmacological preconditioning, with drugs such as cyclosporin, would require the commencement of an additional drug infusion. This would be in addition to a range of infusions that the donor would already be receiving, and so would make little difference to the medical management of the donor. Heat preconditioning requires elevation of the body temperature by several degrees. This could be achieved by the use of warming blankets, increasing the temperature of inhaled gases with which the donor is ventilated, heating intravenous fluids, and possibly the use of heat lamps. Ischaemic preconditioning would be applied when the organs were being removed. This is achieved by commencing the retrieval operation as normal and then, immediately prior to perfusing the donor with cold preservation solution, clamping the blood vessels supplying the liver for ten minutes, and subsequently unclamping them for ten minutes to allow recovery. Perfusion with the cold preservation fluid would then be commenced and the remainder of the organ retrieval procedure performed as normal. Thus the practical implementation of preconditioning treatments ranges from the minor addition of a drug or a modification of operative technique to more interventional options such as whole body hyperthermia.

\section{Clinical precedents}

The optimum management of heartbeating organ donors currently involves the administration of a variety of drugs and infusions to maintain the organs in a suitable condition for transplantation. Preconditioning agents would be used to improve the target organs, rather than merely limiting the detrimental effects of brain stem death. Thus preconditioning crosses the boundary from maintenance to treatment, a fact which could give rise to ethical concerns. There are, however, several precedents for this type of active treatment. Hormonal resuscitation with triiodothyronine, vasopressin, and methylprednisolone is administered to heart transplant donors, ${ }^{13}$ with the aim of correcting the endocrine imbalances that occur after brain stem death. These are active treatments, intended to increase the number of organs suitable for transplantation. They differ from preconditioning, however, in that they represent maintenance rather than therapeutic measures. Thus the boundary between donor maintenance, optimisation, and preconditioning can be considered indistinct.

\section{Clinical application of preconditioning}

There are several applications for preconditioning. Each individual setting requires different considerations with 
regard to both the practical implementation of a preconditioning strategy, and the ethical issues it raises. Donor organs for transplantation currently come from three sources: living donors, brain stem dead heartbeating donors, and nonheartbeating donors. The application of preconditioning strategies raises both ethical and practical issues in each of these three donor groups. Similarly, different methods of preconditioning may be more or less acceptable to families of donors and any treatments used for preconditioning may require lack of objection or assent additional to that obtained for the actual donation.

\section{ETHICAL CONSIDERATIONS \\ Lack of objection/assent}

Consent for organ donation has been a contentious issue in the past. In the UK, even when the potential donor carries an organ donor card, the assent or lack of objection of the next of kin is sought. ${ }^{14}$ The introduction of the Human Tissue Bill may change the emphasis of this process, giving greater priority to the autonomy of the donor. The provision of in depth details of the retrieval process required to explain a preconditioning strategy could potentially lead to a withdrawal of lack of objection to organ donation. Thus describing the preconditioning strategies, or asking for explicit assent to apply them, could potentially have a negative effect on donation rates. This issue must be considered in the light of potential benefits to society as a whole, of advancing science, and improving medical outcomes.

There is an alternative argument that preconditioning represents part of the normal process of organ harvesting and therefore should not require further assent or lack of objection beyond that associated with organ donation itself. Indeed, in his treatise on the ethics of organ donation, Price suggests that "where an individual has requested that his/her organs be used for transplantation after death, it seems correct to infer that permission is granted for procedures which form part of the routine preliminaries to transplantation without seriously compromising the patient in any way". ${ }^{15}$

\section{Law and ethics}

Even where there is no explicit authorisation of procedures for maintaining donors and their organs, they are not necessarily unlawful. Although explicit legislative provision to cover organ preconditioning techniques may be desirable, it is far more important that society endorses the concept that preconditioning strategies in donors to improve organ function and outcome are ethically acceptable and clinically warranted. This would avoid the difficulties that occurred with elective ventilation, which began in the UK in accordance with Home Office directives and agreement, but which has since been abandoned due to legal and ethical concerns. ${ }^{16}{ }^{17}$ Such legislation exists in Sweden, and it states: "Once it has been established that death has occurred, measures may be taken, if appropriate, in order to preserve organs or other biological material pending transplantation... Unless there are special circumstances, such measures should not continue beyond 24 hours" (Price, ${ }^{15} \mathrm{p}$ 170). With regard to organ procurement for transplantation, the UK Human Tissue Bill currently being considered by parliament states that "it shall be lawful...to take steps for the purpose of preserving that part [part of a body] for use in transplantation, and to retain the body for that purpose". However, this authorisation "shall only extend to the taking of the minimum steps necessary...and to the use of the least invasive procedure ${ }^{\prime 18}$ It is unclear whether such authorisation would extend to preconditioning techniques.
The timing of certain preconditioning treatments may be crucial, particularly with regard to agents that may require a significant length of time prior to harvest to provide the beneficial effect. Thus there may be the requirement to commence administration of the agent before lack of objection is obtained. Although this may be against normal principles, this type of approach has previously been approved by ethical committees and has been used in clinical trials on organ donors. ${ }^{19}$ This is also analogous to the management required for uncontrolled non-heartbeating donors where, if the coroner agrees, cooling is commenced as soon as practicable, and then withdrawn if an objection to donation is expressed..$^{20}$ This is provided for in Dutch law, which declares that necessary measures to maintain the organ in a suitable condition for transplantation may be taken after death, so long as the procedure for obtaining the necessary consent has not been completed. ${ }^{21}$

\section{Respect for the donor}

The question of preconditioning having ethical issues derives from the observation that these manipulations will not benefit the individual directly in any way. There is indirect benefit, from the donor's volition to donate organs for transplantation being fulfilled, but is this enough?

Societal beneficence and justice require that the maximum good be obtained from the gifted organs. This could also be seen as an extension of autonomy (albeit posthumously), where respect for the wishes of the deceased to be an organ donor mandates that the transplant procedure be as effective and beneficial as possible. Thus it could be argued that there is a moral and ethical imperative to use preconditioning strategies if they improve the use of donated organs. Autonomy is difficult to establish once an individual has died, but this may become easier as the use of advance directives increases. As has been described in the debate on elective ventilation, however, the lack of public knowledge about specific treatments (such as elective ventilation) means that any impact of advance directives is likely to be limited (Price, ${ }^{15} \mathrm{p}$ 189).

The act of joining an organ donor register or carrying a donor card may be considered an indication of consent to organ donation, but it is not clear whether such consent would extend to preconditioning strategies. There is a need to establish whether preconditioning can be seen as no different from other medical interventions, such as the administration of heparin to donors, and also whether consent to organ donation implies consent to all techniques required to allow good use of the organs. As stated above, justice and societal beneficence require that the best possible use is made of the organs, and therefore that all beneficial manipulations are used.

It is conceivable, however, that the relative importance ascribed to such considerations might alter depending on the nature of the preconditioning strategy considered. It seemsfor example, unlikely that many relatives would object to the deceased donor being given a drug to improve organ function but it is possible that they might raise objection if it were necessary to keep the donor ventilated for a prolonged period of time to allow the drug to take effect. Similarly, more interventional preconditioning techniques, such as whole body hyperthermia, ${ }^{22}$ may be less acceptable to donor families.

\section{SPECIAL CONSIDERATIONS Non-heartbeating donation}

In the controlled non-heartbeating donor, death is not diagnosed until cessation of cardiac function. In this setting interventions prior to death, either to optimise the donor or to precondition organs, may alter the process leading to death 
and this is considered unacceptable. The guidelines currently being drawn up by the Intensive Care Society state quite clearly that "It is inappropriate to escalate current treatment, add new therapies-for example, inotropes, heparin, hormone replacement, or to undertake invasive interventionsfor example, vascular cannulation before death for cold perfusion, to improve organ viability". ${ }^{23}$ Preconditioning in this setting is only potentially possible after the diagnosis of death and an acceptable period (frequently 10 minutes) during which no intervention is made on the potential donor. In terms of practicality this means that only preconditioning strategies that involve perfusion of the deceased donor or involve ex vivo perfusion of organs would be acceptable in this context.

\section{Living donation}

Living donors provide the simplest model, where the donor is fully aware of the donor procedure and can provide detailed consent, with prolonged time for consideration and reflection. This situation therefore poses few ethical considerations not encompassed by fully informed consent. Although all organs donated for transplantation are considered precious, the act of living donation places additional responsibilities on the medical profession. Living donation of organs or tissues is one of the few circumstances in medicine where the donor undergoes a procedure with no physical benefit to themselves. In this context it is imperative that the risk to the donor is minimised. Similarly, because there is a risk to the donor, however small, it is generally accepted that the donated organ should have a reasonable chance of successful transplantation. Considering these issues, any preconditioning strategy applied to a living donor population must not only demonstrate benefit to the recipient but must also not place any additional burden or risk on the donor.

\section{Multiorgan donors}

The majority of organ donors in the UK donate more than one organ. This raises the question whether, if a preconditioning strategy had been demonstrated to confer a beneficial outcome for a particular organ, it would be acceptable to use such a treatment in a multiorgan donor if the effects on other organs were not known. The answer in this case would probably be negative and so research involved in the development of such strategies should consider effects on other organs that might be donated simultaneously in a clinical setting. If the effects of a treatment on other organs were not adverse, the question would then arise as to whether the potential recipients of other organs should be consulted and requested to give their consent to preconditioning being used. The issues of consent and collateral involvement become more prescient in the context of a clinical trial where the effect of a preconditioning strategy may be the subject of evaluation.

\section{Recipients of preconditioned organs}

In many circumstances where preconditioning would be used it may be impractical to obtain consent from the recipients in time to influence the application of the preconditioning intervention. There is concern therefore that if a recipient objects to receiving an organ that has undergone preconditioning this may subvert the normal informed consent process by placing coercion on the recipient. Thus the desire to receive a transplant may outweigh the concerns or objections of the recipient to receiving an organ from a research protocol. Adopting a utilitarian approach, it could be argued that preconditioning has the potential to improve the outcomes of transplantation and that society should support research which aims to advance science or improve outcomes in medicine. Following this argument to its practical application, the occasional refusal to receive a preconditioned organ would be offset by the perceived benefit to society as a whole. In practice perhaps the easiest way to deal with this issue would be to inform patients, at the time of addition to the waiting list, that they may be offered organs derived from trials of preconditioning protocols and that they should consider whether they would accept such an organ. They should also be informed that such protocols had received approval from local regional ethics committees and that such approval would not normally have been granted if there were issues over either safety or perceived benefit.

\section{Preconditioning in a research setting}

In common with other medical research, trials of preconditioning should abide by the principles enshrined within the Declaration of Helsinki. ${ }^{24}$ Specifically there should be an adequate laboratory and animal research base to support human studies and these studies should have clear potential for benefit counterbalanced against the inherent risk of the intervention. The design of studies should be clearly set out and be undertaken by competent individuals. Studies should have been reviewed and approved by an independent committee-in the UK this would be the local or multicentre research ethics committee, as appropriate. Articles 10 and 11 of the Declaration of Helsinki deal with areas of research where preconditioning could raise ethical issues. Article 10 relates to the situation where an individual may be in a dependent relationship with the researcher, a possibility for the recipient of an organ used in a preconditioning study, and article 11 describes the case where the individual is legally incompetent-the situation of the deceased donor. These issues present specific problems for regional ethics committees, which often have to consider them without precedent for comparison or using guidelines derived from research in living subjects.

\section{CONCLUSION}

Preconditioning strategies have a great potential to improve outcome in solid organ transplantation and also to increase the availability of organs by allowing use of marginal donors. As with many issues in transplantation, there are few precedents to provide guidance on the practical implementation of such programmes. There are likely to be moves to integrate these treatments into organ procurement protocols in the near future. There is, therefore, a clear need to establish an ethical consensus on the acceptability of such treatments and the responsibilities of those involved in their administration to donors, their families, and recipients. It is hoped that transparency in such discussions will both preempt misunderstandings arising from perceptions of unnecessary intervention or experimentation on the deceased donor and provide an ethically acceptable framework upon which preconditioning strategies could be based.

\section{ACKNOWLEDGEMENTS}

SJM and EMH are funded by the Scottish Hospital Endowments Research Trust (SHERT). SJW is funded by the Wellcome Trust.

\footnotetext{
Authors' affiliations

S J McNally, E M Harrison, S J Wigmore, Tissue Injury and Repair Group, MRC Centre for Inflammation Research, Medical School, University of Edinburgh, Edinburgh, UK

Competing interests: The authors all hold grants and work in the field of experimental preconditioning. SJM and EMH are funded by the Scottish Hospital Endowments Research Trust (SHERT). SJW is funded by the Wellcome Trust and is a member of the ethics committee of the British Transplantation Society, although this article has been written independently of this association and is not intended to represent either the views of the ethics committee or the society as a whole.
} 
Correspondence to: Stephen McNally, Tissue Injury and Repair Group, MRC Centre for Inflammation Research, Medical School (6 floor), University of Edinburgh, Teviot Place, Edinburgh EH8 9AG, UK; sj.mcnally@ed.ac.uk

Received 12 December 2004

In revised form 18 January 2005

Accepted for publication 22 February 2005

\section{REFERENCES}

1 Lazzaretti CT, Carvalho JG, Mulinari RA, et al. Kidney transplantation improves the multidimensional quality of life. Transplant Proc 2004;36:872-3

2 More transplants - new lives. Transplant activity in the UK 2003-4. Bristol: UK Transplant, 2004.

3 Koti RS, Seifalian AM, Davidson BR. Protection of the liver by ischemic preconditioning: a review of mechanisms and clinical applications. Dig Surg 2003;20:383-96.

4 Cleveland JC Jr, Raeburn C, Harken AH. Clinical applications of ischemic preconditioning: from head to toe. Surgery $2001 ; 129: 664-7$.

5 McLaren AJ, Friend PJ. Trends in organ preservation. Transpl Int 2003; 16:701-8.

6 Kosieradzki M. Mechanisms of ischemic preconditioning and its application in transplantation. Ann Transplant 2002;7:12-20.

7 Clavien PA, Selzner M, Rudiger HA, et al. A prospective randomized study in 100 consecutive patients undergoing major liver resection with versus without ischemic preconditioning. Ann Surg 2003;238:843-50.

8 Redaelli CA, Wagner M, Kulli C, et al. Hyperthermia induced HSP expression correlates with improved rat renal isograft viability and survival in kidneys harvested from non-heartbeating donors. Transpl Int 2001;14:351-60.

9 Terajima H, Thiaener A, Hammer C, et al. Attenuation of hepatic microcirculatory failure during in situ xenogeneic rat liver perfusion by heat shock preconditioning. Transplant Proc 2000;32:1111.
10 Yang CW, Ahn HJ, Han HJ, et al. Pharmacological preconditioning with low dose cyclosporine or FK506 reduces subsequent ischemia/reperfusion injury in rat kidney. Transplantation 2001;72:1753-9.

11 Camargo CA Jr, Madden JF, Gao W, et al. Interleukin-6 protects liver against warm ischemia/reperfusion injury and promotes hepatocyte proliferation in the rodent. Hepatology 1997;26:1513-20.

12 Chauveau C, Bouchet D, Roussel JC, et al. Gene transfer of heme oxygenase-1 and carbon monoxide delivery inhibit chronic rejection. Am J Transplant 2002;2:581-92.

13 Rosendale JD, Kauffman HM, McBride MA, et al. Aggressive pharmacologic donor management results in more transplanted organs. Transplantation 2003;75:482-7.

14 UK hospital policy for organ and tissue donation. Bristol: UK Transplant 2003.

15 Price D. Legal and ethical aspects of organ transplantation. Cambridge University Press, 2000:211.

16 Shaw AB. Non-therapeutic (elective) ventilation of potential organ donors: the ethical basis for changing the law. J Med Ethics 1996;22:72-7.

17 Riad H, Nicholls A, Neuberger J, et al. Elective ventilation of potential organ donors. BMJ 1995:310:714-18.

18 UK Human Tissue Bill 2004: section 44: preservation for transplantation: subsections 1 and 2 .

19 Guesde R, Barrou B, Leblanc l, et al. Administration of desmopressin in brain dead donors and renal function in kidney recipients. Lancet 1998:352:1178-81.

20 Brook NR, Nicholson ML. Kidney transplantation from non heartbeating donors. Surgeon 2003;1:311-22.

21 The Organ Donation Act 2003. The Hague: International Publication Series Health Welfare and Sport no 3, 2003, section 22:subsection 2.

22 Mokuno Y, Berthiaume F, Tompkins RG, et al. Technique for expanding the donor liver pool: heat shock preconditioning in a rat fatty liver model. Liver Transp/ 2004; 10:264-72.

23 Intensive Care Society. Draft guidelines on organ donation. London: Intensive Care Society, 2004, http://www.ics.ac.uk/downloads/Standards/ Master\%20ICS\%20Guidelines\%20all\%20sections\%20(Nov04).pdf (accessed 12 Apr 2005).

24 World Medical Organisation. Declaration of Helsinki. BMJ 1996;313:1448-9.

\section{Notice}

Increasing ethics, communication, and social science content for written exams in undergraduate medicine

Hosted by the Universities Medical Assessment Partnership (UMAP), this is a workshop to disseminate good practice in question writing whilst also helping to incorporate ethics, communication, and social science questions into the UMAP bank. This will serve to encourage these topics to be assessed at UMAP partner medical schools who at present include Newcastle, Leeds, Liverpool, Manchester, and Sheffield.

Date: Thursday $24^{\text {th }}$ November 2005

Timings: Workshop 11.00 - 1.30pm; Lunch 1.30pm; Workshop $2.30-5.00 p m$

Place: Gartree and Rutland, $4^{\text {th }}$ Floor, Charles Wilson Building, Leicester University

Presenter: Andrea Owen, UMAP Project Manager

Places are free of charge and can be booked by contacting the UMAP office by email, umap@fs1.with.man.ac.uk or telephone, 0161291 5805. See the project website for more details www.umap.man.ac.uk 